\title{
慈育大学中文系学生综合课听写现状分析
}

\section{ANALISA KONDISI DIKTE MAHASISWA PELAJARAN KOMPREHENSIF PROGRAM STUDI BAHASA DAN BUDAYA TIONGHOA UNIVERSITAS BUNDA MULIA}

\author{
Kumala Dewi ${ }^{1 ;}$ Timotius Kirana Karya ${ }^{2}$ \\ Universitas Bunda Mulia \\ Email: kdewi@bundamulia. ac.id
}

\begin{abstract}
摘要
本次研究针对于慈育大学中文系一至四年级的学生, 总有 165 名, 分别是 5 名教师 与 160 名学生。调查方式主要通过问卷及学生的听写本, 通过问卷得知学生综合课的听 写状况, 包括学生对听写的看法与兴趣、学生听写前后的做法与听写时遇到的障碍, 教 师进行听写的方法、听写时的做法、以及听写后的做法。通过听写本探讨学生的错别字。
\end{abstract}

关键词：听写现状; 汉语综合课; 错别字; 慈育大学中文系

\section{一 引言}

汉字有着悠久的历史, 至今已有 5000 多年的历史了。它的结构成分都是通过一个 或两个以上的笔画来合成一个字, 因此在学习书写汉字的实践过程中, 学习者必须掌握 汉字的部件和笔顺。除此之外, 汉字作为最古老的连续使用的书写文字使得它的总数非 常多。每个汉字都有一定的读音, 但是有的具有同一个字形记录了不止一个读音和意义; 有的具有同一个读音却可以用多个字形记录。这些汉字的特点使得汉字作为世上最难写 的文字, 也使对外汉语学习者遇到不少的困难, 尤其是在书写汉字以及把它死记硬背。 因此, 学习者必须反复练习, 才能牢记每一个字。

为了提高学生的书写能力及对汉字的记忆能力, 教师一般都会采取基本的传统措 施, 即 “听写”。大部分的听写都是在综合课上, 而慈育大学中文系的综合课, 没一个 年级均有, 但因为不同的教师会有不同的听写方式与内容。学生又对哪一种方式感兴趣, 哪一种学习方式最有效, 我们想通过本次研究得出结果, 进行讨论与分析。除此之外, 我们也会将学生的听写本收集起来, 将学生的错字进行分析。希望通过本次的研究结果 
可以为教师提供有效的听写建议，也为学生提供有意义，有参考价值的文献。

\section{1 学习焦虑的定义}

听写作为外语教学手段已有悠久的历史。蒋丽萍在《汉语作为第二语言课堂教学》 指出, 听写是训练学生听和写的配合能力。教学时教师以一定的口述内容, 学生边听边 写, 它需要学生的耳、眼、手、脑并用, 是训练学生掌握字词句的有效方法。杨寄洲在 《对外汉语课堂教学技巧》也有提到关于听写。听写是一个非常重要的语言课堂教学练 习项目, 也是汉字教学中非常重要的一种练习形式, 学生听教师或录音的声音, 把听到 的内容 (词语、句子、短文, 通常包括声调) 用汉字写下来。它由声入手, 训练学生听 到字音之后联想汉字，最后学生独立地书写出正确的汉字。

总而言之, 听写是一种课堂练习手段, 能测试学生的语言项目, 尤其是学生的听力 和书写能力, 此外也训练学生的短时记忆能力。因为听写练习要求学生把听到的内容很 快地用文字记录下来。

\section{2 听写的过程}

根据听写内容和要求的不同, 张辉和杨楠在《汉语综合课教学法》把听写分成以下 七个类型:

1) 听写声母

测试方法：学生听音节, 并用拼音记录所听到音节的声母。

例: 听: xuéxí

看: _ué_í

写: $\underline{x}$ ué $x \underline{i}$

2) 听写韵母

测试方法：学生听音节, 并用拼音记录所听到音节的韵母。

例: 听: xuéxí

看: X

写: x ué x $\underline{1}$

3) 听写声调

测试方法: 学生听音节, 并用拼音记录所听到音节的声调。

例: 听: xuéxí

看：xuexi 
写: xuéxí

4) 听写音节

测试方法: 学生听音节, 并用拼音记录所听到的音节。

例: 听: xuéxí

写: _xuéxí

5) 听写句子，写拼音

测试方法: 学生听句子, 并用拼音记录所听到的句子。

例: 听: Wŏ zài Běijīng xuéxí Hànyŭ.

写: Wŏ zài Běijīng xuéxí Hànyŭ.

6) 听写句子

测试方法: 学生听句子, 并用汉字记录所听到的句子。一般每个句子听三遍, 第一 遍要求听懂, 第二遍要求边听边写, 第三遍要求边听边检查书写是否正确。

例：你的房间太乱了，把这些乱七八糟的东西收拾一下吧。

7) 听写语段

测试方法: 学生听语段, 并用汉字记录所听到的语段。一般听三遍, 第一遍 整段听, 要求听懂; 第二遍分句听, 要求边听边写; 第三遍再整段听, 要求边听边检查 书写是否正确。

例: 我对自己的工作很不满意, 打算辞职。朋友建议我先把公司的一切贸易技巧完 全搞通再辞职不干。除了弄清楚写商业文件的方法以外, 还有学会熟练地操作电脑。我 听从了朋友的建议, 从此刻苦学习, 甚至下班以后, 仍然留在办公室加班。一年之后, 我发现老板对我刮目相看, 我已经成了公司的红人了。

\section{3 听写的作用}

杨寄洲在《对外汉语课堂教学技巧》提出了听写的作用, 主要是:

1) 听写能练习语音的技巧

语音练习可分为两大类: 一类是发音练习, 一类是听音练习。在语音教学阶段, 发 音和听辨是相辅相成的两个方面, 模仿只是形成汉语发音习惯过程的一部分, 其前提是 要有正确的听辨能力, 否则是模仿不好的。通过听写可以检查学生辨别声、韵、调和音 节、句子的能力，同时可以熟悉汉语拼音的拼写规则。

2) 听写能展示语法点技巧 
展示语法点是语法教学的第一步。展示得法, 可以减少学生在理解上的难度, 理解 所学语法点的用法。听写是最常见的一种展示语法点的方法。常在上课时配合检查学生 复习和预习的情况时使用。教师可以选择两三个例句, 让学生写下来。比如要展示语法 点动词“完”作结果补语时, 让学生写: 我们学完二十三课了。

3) 听写能练习汉字的技巧

汉字练习是指为了使学生达到正确认读、书写及灵活运用汉字而进行的各种形式的 练习。通过听写可以用来检查已经学过的汉字, 也可以用来检查前一课布置的预习内容, 也可以用来作为引入新课程的手段。听写有多种形式, 如听写单字、词语、句子或成段 的章节。初级可以让学生在汉字旁加注汉语拼音, 以了解学生对汉语拼音的掌握情况。 除此之外, 听写还可以综合训练学生对字的形音义的记忆。只有多写多练才能加深学生 对汉字的印象, 达到真正的掌握。

\section{4 汉字书写错误}

孙德金、朱志平和冯丽萍提出了错别字大致有如下几种的类型 :

1) 笔画增损:

如: 将“慕”字下部多加一点, 将“美”字少写一横。

2) 笔形失准:

如：将“谢”字中的部件“寸”写作“才”，将“切”中的的部件“七”写作“土”。

3) 结构错位:

如: 将“多”字的两个部件“夕”横向排列。

4) 形近混淆:

如: 司 $\leftrightarrow$ 可 庆↔厌 鸟 $\leftrightarrow$ 乌 看

a. 特别是意符同而声符形似的字如: 绿 $\leftrightarrow$ 缘 没 $\leftrightarrow$ 沿

b. 或者是声符同而意符形似的字如：辨 $\leftrightarrow$ 辩 $\quad$ 侍 $\leftrightarrow$ 待

5) 音同混淆

如: 常 $\leftrightarrow$ 长 在 $\leftrightarrow$ 再 作 $\leftrightarrow$ 做 围↔ 维

6) 繁简误推:

如将“积”的右部写作“责”, “运”的上部写作“军”。

7) 部件错误: 
a. 部件错位, 如: 把“欢”的两个部件调换了位置, 把“没”的右侧部件上下调换了位 置。

b. 部件缺失或者部件增加, 如: 把“供”写成“共”, “响”写成“向”; 再比如“门”写成“间”, 或者“们”写成“门”，等等。

\section{二 听写总体状况}

\section{1 对听写的看法与兴趣}

从调查问卷得出的结果看, 无论学生还是教师都一致认为听写很重要。从教师的角 度来看, 认为听写可以检查学生的预习情况、训练学生的听辨能力, 以及训练学生的书 写能力, 所以很多教师在课堂上都会进行听写。从学生的角度来看, $78 \%$ 的学生认为听 写能训练他们的书写能力, 通过听写可以让他们记住生字, 可以背生词、增加自己的词 汇量以及训练自己的记忆力。

虽然学生都知道听写的作用是什么并且认为听写是重要的, 但对听写的兴趣并不 高, 有的学生还会把听写当成压力, 有听写就意味着他们必须学习。从得出的结果来看 超过一半的学生对听写不是很感兴趣, 只有 $22.5 \%$ 的学生对听写有兴趣, $65.6 \%$ 的学生 则感到 “一般” ，其余的学生对听写并不感兴趣。

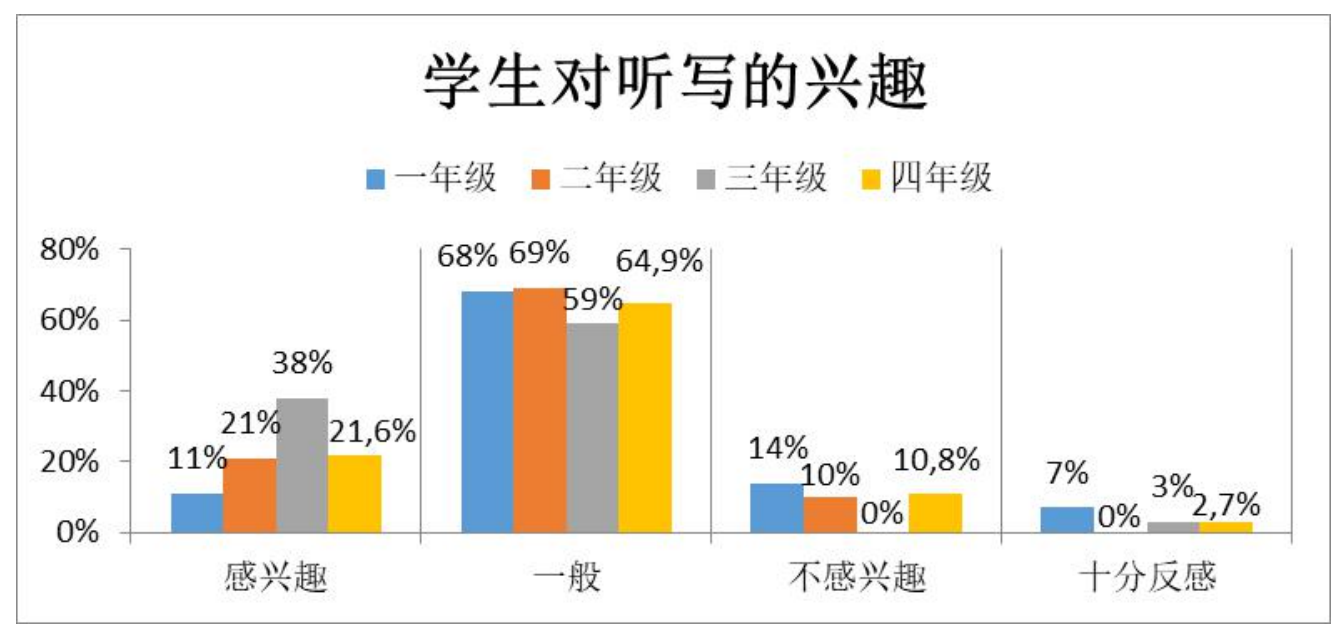

从以上的结果我们可以总结出, 兴趣与看法并不相关, 学生一致都认为听写 很重要, 也肯定了听写带来的好处, 但对听写的兴趣却不是很高。 


\section{2 对听写的看法与兴趣}

为了得知教师的听写方式, 我们也将问卷发给教师, 从问卷结果来看, 有 $80 \%$ 的教 师使用 “教师念, 学生写” 的方式进行听写, 也就是最基本, 最常用的听写方式, $20 \%$ “自愿到黑板前听写”。从学生的问卷结果得出, 学生对这样的听写方式并不是很感兴 趣, 超过一半也就是 $69.4 \%$ 的学生都选择了 “一般” 为答案。学生对一成不变的听写方 式, 觉得单调、不够多样化。虽然对听写方式不是很感兴趣, 但又觉得听写对训练他们 书写汉字的能力有帮助, 所以学生还是会因为有听写而努力学习。

从得出的结果, 学生对听写本身不是很感兴趣, 对教师的听写方式也不是很感兴趣, 但一问到学生理想的听写方式, 占 $64.4 \%$ 的学生都认为还是按常规的听写方式进行听写, 也就是 “教师念、学生写” 。这说明学生虽然不是很感兴趣, 但又认为常规的听写方式 也是最好的方式了。另外有 $24.4 \%$ 的学生希望教师进行一个有兴趣性的听写方式, $11.3 \%$ 选择了自愿到黑板上听写生词。

\section{对听写方式的兴趣与理想的的听写方式}

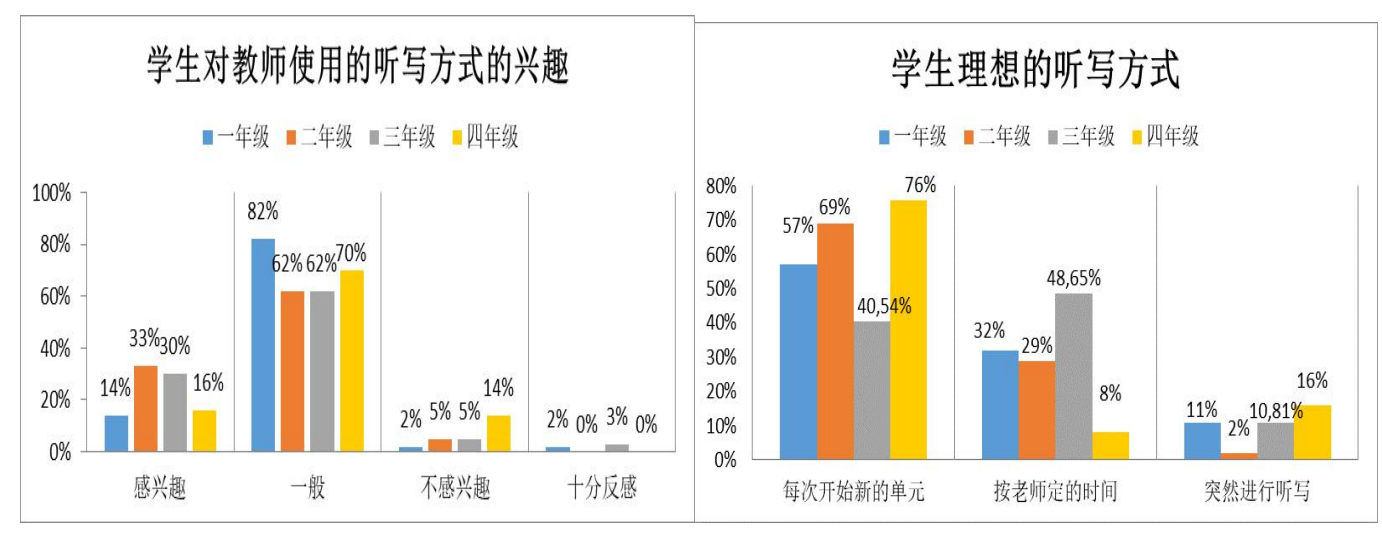

从听写的时间来看, 超过一半的学生希望每次开始新单元进行听写, $29.3 \%$ 学 生认为按教师规定的时间听写, $10 \%$ 的学生则认为突然进行听写。

\section{3 教师进行听写的方法}

教师大部分用课堂 10-20 分钟的时间进行听写, 慈育大学一次课 100 分钟, 也就是 说占了 $10 \%-20 \%$ 的上课时间, 占用的时间相当于课堂导入的时间。我们认为 10-15 分钟 的时间比较合理, 因为综合课要讲的内容非常多, 因此不能占用太多的时间用来听写。 


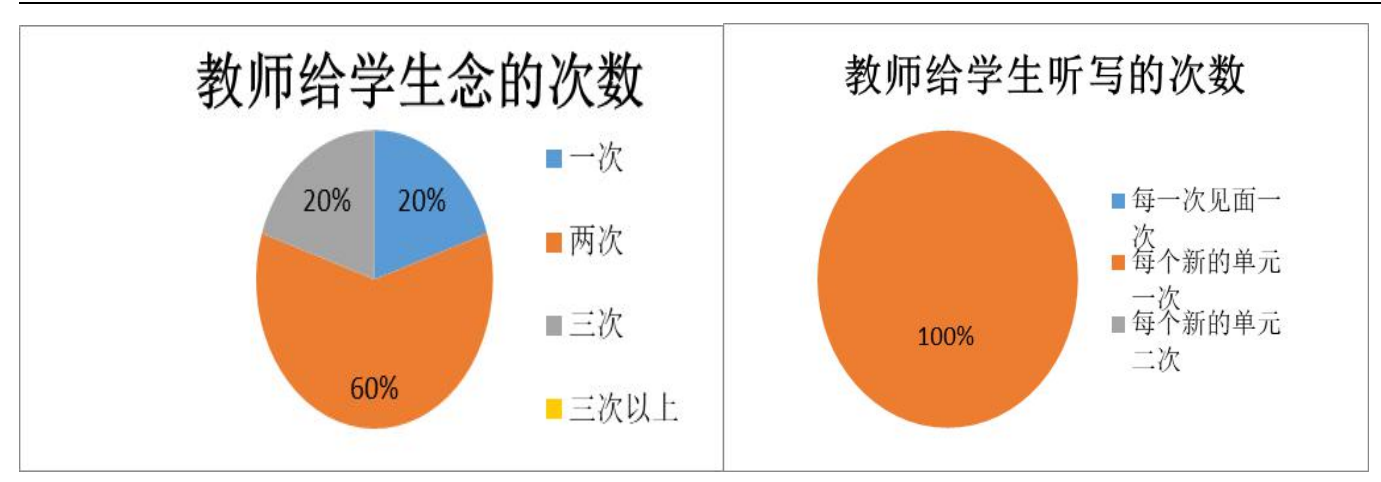

$100 \%$ 的教师在每一个新单元听写一次，使用的内容 $100 \%$ 来自于生词表，只有一个 教师采用课文内容。在进行听写的时候教师会念两次, 要是念的时候有学生没听清楚的 话, 还会再念一次。一般来说要是学生听一次就能写出来了, 那就说明学生已经学习了, 有的学生没听清楚会要求教师再念一次, 这是合理的。如果学生没有学习无论教师念多 少次也难以写得出来, 因此我们认为念两次是最合理的, 要是还是有学生没听清楚, 念 第三次, 再不清楚就只能说明学生听力能力不好或者没有学习。

\section{4 学生听写的准备情况}

据我们了解教师没有布置听写学生就不学习, 没有听写学生就不去学习生词表的生 字, 另外使他们不学习的原因可能是除了综合课还有其他的课要准备, 还有很多作业要 完成。

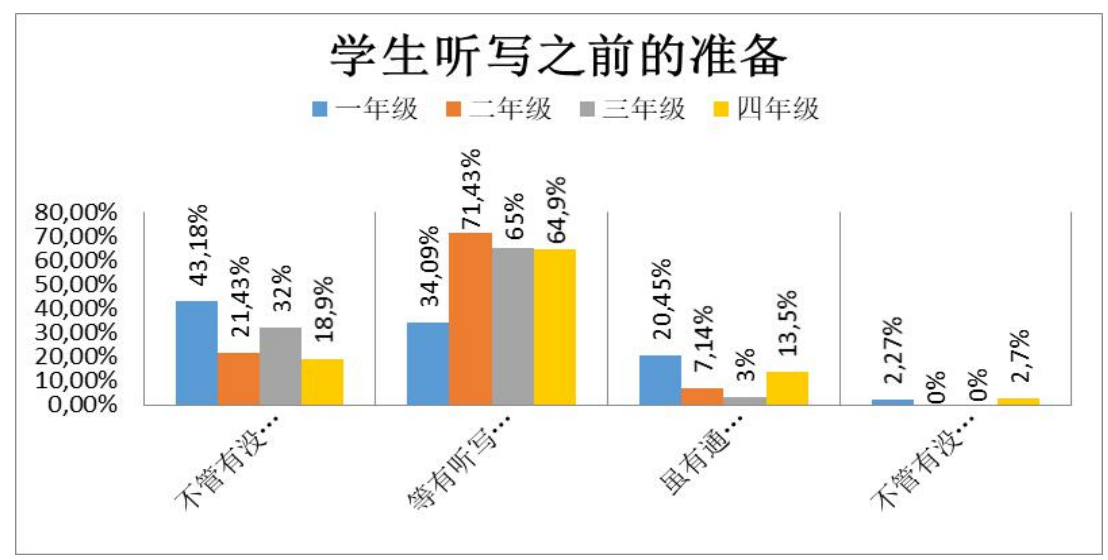

上述的表可以证明我们做调查之前的探讨, 学生要等有听写才会学习占 $58.1 \%$ 就 是占了超过一半的人数, 12.6 的学生无论有没有听写都很少做准备甚至没有准备, 当然 有的学生非常积极, 无论有没有听写都会自动学习, 160 个学生里有 47 个这样的学生, 占 $29.4 \%$ 的总人数。在我们看来有将近 $30 \%$ 的积极学生, 这个数目已算是乐观了, 因为 这些学生有自主学习的能力, 对自己的要求高, 汉语水平也会因此提高。老师可以用其 
他活动来刺激学生的自主学习, 让他们意识到不学习就很难取得好成绩, 不能只有考试 的时候才学习。

\section{5 学生听写的主要状况}

学生听写的时候常常会写不出来, 教师念的时候有时候反应不过来, 原因各不相同。 觉得汉字难写难背的学生占 41.9, 这也就是学生的最大困难, 一年级的学生生词还不是 很难, 但二年级、三年级、四年级课本的生词表里面汉字非常多, 也特别多四字成语, 这给学生造成了困扰与难题。其二是听不清楚, 可能老师念的声音太小, 学生听不清楚, 占总数的 $21.9 \%$, 而四个年极当中三年级的学生占 $46 \%$, 三年级的生词表里面成语非常 多, 这容易让他们把生词混淆, 没听清楚写错字。

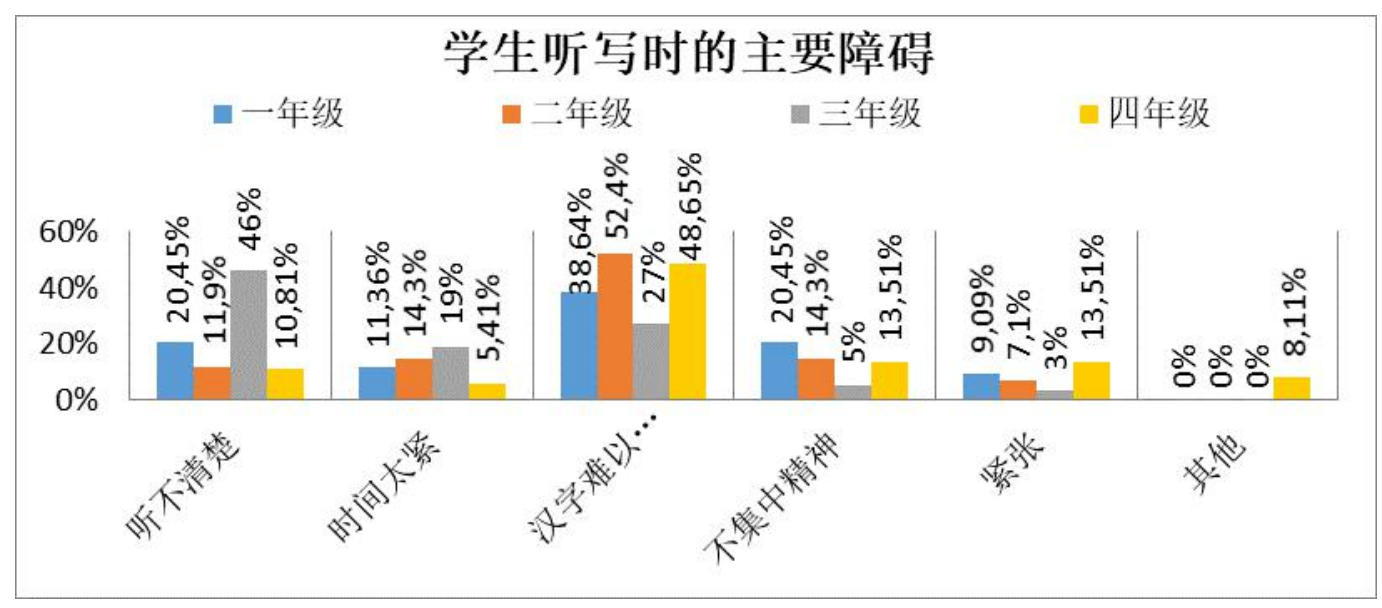

使学生写不出来的主要原因不仅仅使听不清楚, 有 $78 \%$ 也就是有 124 个学生表示 听写时忘记怎么写成了他们最主要的原因, 除了容易把生词混淆, 生词太多也让学生难 以记住, 难以背下来, 听写时虽然听得很清楚但就是写不出来。写不出来的时候有超过 一半也就是 $59.4 \%$ 的学生用拼音写, 虽然教师看到拼音也不会给分数, 学生还是会把空 白的地方天上拼音。另外常出现的错误就是学生常常用同音词来代替他们不会写的汉 字, 虽然知道写上去的汉字是错的字, 但学生还是会这样做。

\section{6 教师对听写之后的做法}

这里主要是要探讨教师批改学生听写本的时候是否会给学生改正错字, 做解释以及 给惩罚。从得出的结果来看, 五个教师当中有 4 教师会改正学生的错字, 只有一个教师 
表示很少。改正学生的错字, 可以让学生知道为什么会写错。从得出的结果来看我们可 以总结出, 教师一般都会改正学生的错字, 但很少给学生解释正确的书写方法, 只有两 个教师表示经常。

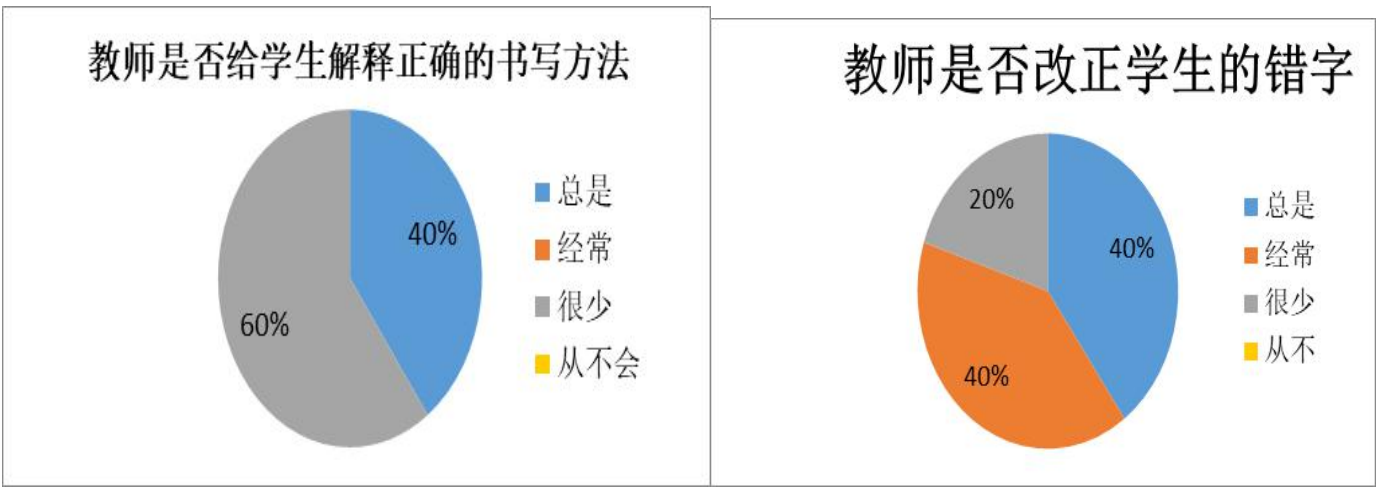

我们认为给学生解释正确的书写方法很重要, 剧我们的了解学生写错可能是看不清 错怎么写, 另外水平差的学生有的字真的不会写, 或者不知道措在哪儿, 让他们改正的 时候还是写出错的字来。因此, 我们认为教师应该给学生解释正确的书写方法, 尤其是 错的频率高的字。而对于改正学生的错字，我们认为教师可以用画圈指出学生的错字， 然后在课堂上发给学生的时候, 先解释给学生正确的书写方法, 再让学生自己改正, 改 正之后再交给教师查看。这样教师省去了不少时间, 学生也认识到自己的错误并知道该 怎么改正错误。

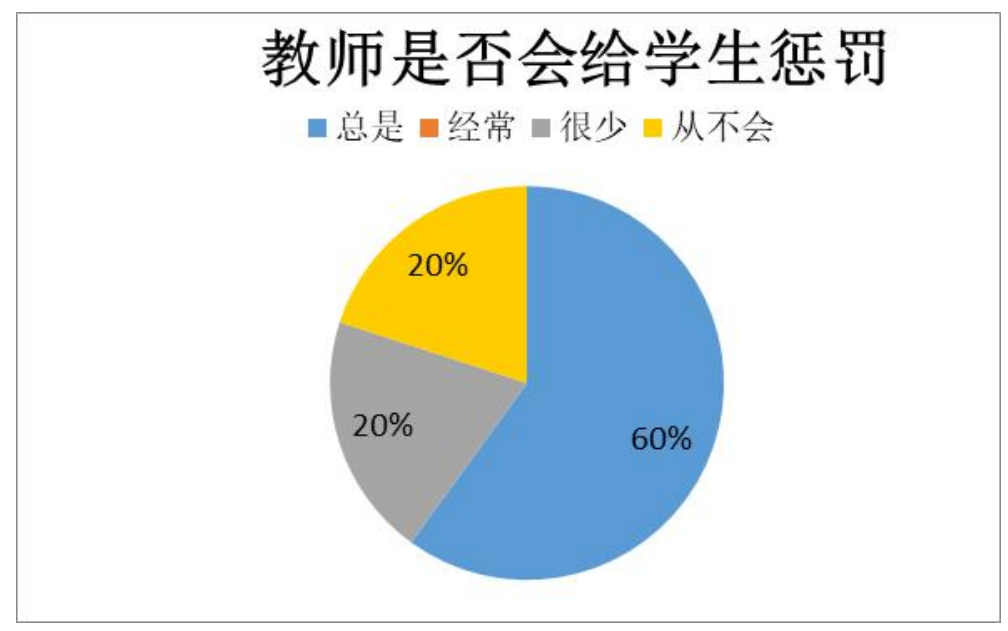

教师们为了让学生记住生词, 让他们听写之后, 对于他们不会写的字一般会给学生 一些惩罚, 有三个教师表示经常给惩罚, 而两个教师表示很少或从不给惩罚。教师给的 惩罚大部分是让学生重写几遍。这样学生就会对自己犯下的错误有印象, 以后就不会犯 同样的错误了。 


\section{三 学生听写时出现的错别字}

\section{1 一年级的错别字}

一年级错字类型统计结果

\begin{tabular}{|c|c|c|c|}
\hline \multicolumn{4}{|c|}{ 一年级 } \\
\hline 写错的人数 & 写错的字 & 错的原因 & 错误类型 \\
\hline 12 & 南 & 把下面的写成 “半” & 笔形失准 \\
\hline 10 & 普 & 上面只写一个点 & 笔画增损 \\
\hline 9 & 冬 & 下面的“捺” 部分写成“敉” & 笔形失准 \\
\hline 8 & 暖 & 把 “目” 写成 “日” & 笔画增损 \\
\hline 4 & 通 & 把 “角” 写成 “角" & 笔形失准 \\
\hline 3 & 竟 & 少了一横 & 笔画增损 \\
\hline 3 & 直 & 中间少了一横 & 笔画增损 \\
\hline 3 & 嗽 & 把 “欠” 写成 “文” & 形近混淆 \\
\hline 3 & 算 & 中间少了一横 & 笔画增损 \\
\hline 3 & 冒 & 上面的部分写成 “曰” & 笔形失准 \\
\hline 3 & 迹 & 把 “亦” 的坚提，写成坚 & 笔形失准 \\
\hline
\end{tabular}

一年级的错字不是很多, 是因为他们听写的比较少, 不是每一个单元都听写, 因此 学生写错的字并不是多, 但有的字写错的人却很多。写 “暖”、“冬”、“普”、“南”时一 年级学生写错的人数超过 5 个人, 那就说明这些字是学生比较容易写错的字, 教师在教 学生写字的时候可以多注意这些字。从错字类型来看笔画增损与笔形失准比较多。 


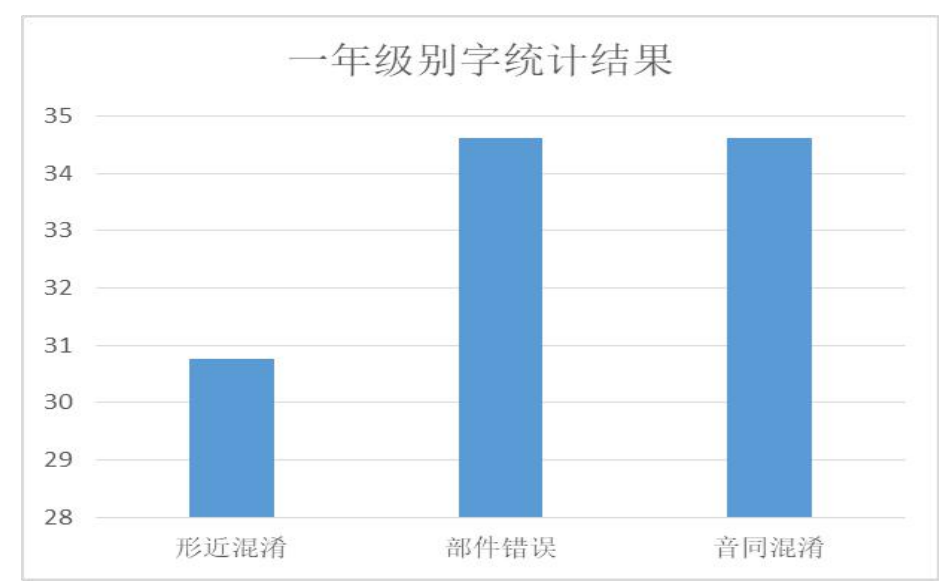

从一年级学生的错别字统计结果来看, 错字大部分写错的原因是笔画失准及笔形相 近, 这可以说明一年级学生刚学习学汉字的时候, 对于笔画与笔形还不是很了解, 所以 常常会因此写错字。而别字方面是形近混淆、部件错误、音同混淆这三个类型甚多。

从错别字的统计来看, 一年级学生对笔画、部件的掌握度还不够高, 使他们在写字 的时候容易写错, 容易把看起来很想的字混淆了。教一年级的教师要特别强调笔画与部 件的教学, 让学生分辨相近的笔形, 或部件, 让他们了解到写字的时候一定要写得正确, 才不会出错, 才不会把字写成错别字。

\section{2 二年级的错别字}

\begin{tabular}{|c|c|c|c|}
\hline \multicolumn{5}{|c|}{ 二年级 } \\
\hline 错的人数 & 写错的字 & 错的原因 & 错字类型 \\
\hline 24 & 讶 & 把 “牙” 写成 “乐” & 笔形失准 \\
\hline 22 & 微 & 把 “父” 写成 “又 “ & 形近混淆 \\
\hline 16 & 冒 & 上面的部分写成 “曰” & 形近混淆 \\
\hline 14 & 悠 & 把 “父” 写成 “又 “ & 形近混淆 \\
\hline 10 & 卑 & 把撇写成坚 & 笔形失准 \\
\hline 9 & 革 & 把上面写成 “+” & 形近混淆 \\
\hline 8 & 观 & 把 “见” 写成 “贝” & 形近混淆 \\
\hline
\end{tabular}




\begin{tabular}{|c|c|c|c|}
\hline 8 & 虑 & “七” 的交叉结构写成连接结构 & 笔形失准 \\
\hline 8 & 勤 & 把上面写成 "+" & 形近混淆 \\
\hline 7 & 融 & 把 “虫” 写成 “犬” & 笔形失准 \\
\hline 7 & 痛 & 把 “角” 写成 “角” & 形近混淆 \\
\hline 7 & 怨 & "夕" 多了一捺 & 笔画增损 \\
\hline 6 & 猫 & 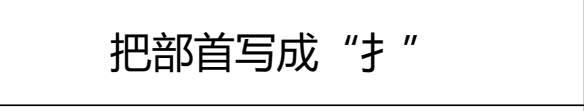 & 笔形失准 \\
\hline 6 & 朗 & 把前边的写成 “民 & 笔画增损 \\
\hline 6 & 伤 & 把“力“写成” 刀“ & 笔形失准 \\
\hline 5 & 狼 & 把部首写成 “き” & 笔形失准 \\
\hline 5 & 烈 & “夕" 上面少一横 & 笔画增损 \\
\hline 5 & 深 & 上面多 “.” & 笔画增损 \\
\hline 5 & 横 & 把上面写成 “+” & 形近混淆 \\
\hline 5 & 尊 & 中间少了横 & 笔画增损 \\
\hline 5 & 察 & 把下面的写成 “夕" & 笔画增损 \\
\hline 5 & 害 & “丰” 与 “口” 没有连在一起 & 笔形失准 \\
\hline 5 & 契 & 把 “刀” 写成 “力” & 笔形失准 \\
\hline
\end{tabular}

上述的结果可以告诉我们二年级的学生常犯的错误类型是笔形失准、形近混淆以及笔画 增损。与一年级对比, 二年级学生的错误并不只是关于笔形与笔画, 而是多了一个类型, 就是 “形近混淆”, 23 个错误里面有 8 个是 “形近混淆”。这可以说明, 一年级的时候 写错字是因为不够掌握笔画与笔形, 到了二年级学习任务重了, 认识的汉字多了, 所以 写字的时候常常把看起来很像的字混淆了。要是学生在学习的时候, 没有仔细学习, 要 掌握的汉字越来越难, 越来越多, 所以很容易让学生把形近的汉字混淆。 


\section{二年级别字类型统计结果}

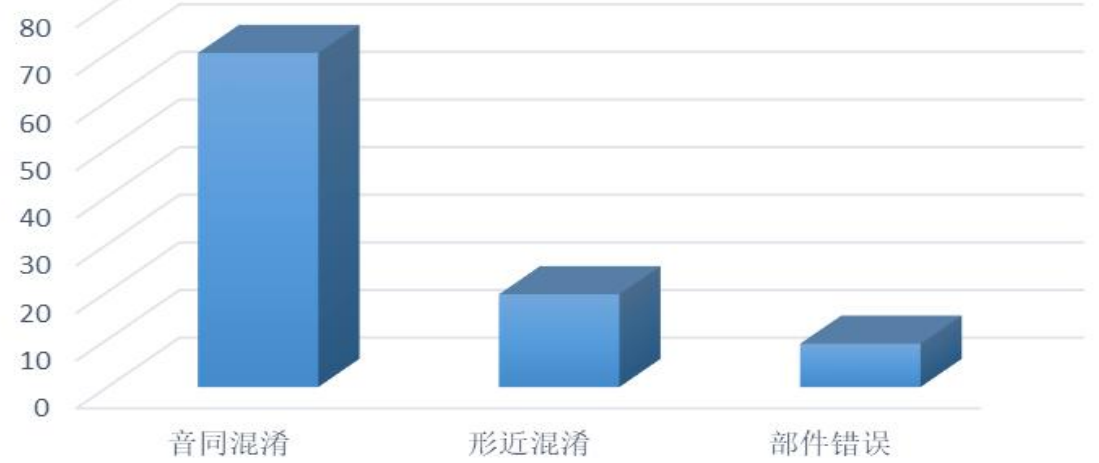

上述的统计结果来看, 结果虽然与一年级别字类型相同, 但结果却不一样, 一年级 的三个类型错误的数量差不多, 而二年级确是音同混淆大大超过与形近混淆与部件错 误。因此我们认为教二年级的时候不在像一年级要强调笔画与部件, 对于二年级更多的 是词语教学, 词的搭配。让学生弄懂, 不同的词有不同的搭配, 虽然有的字读起来一样 但搭配的字却是不一样, 就如上述表里的字, 错误频率超过 5 的字有 15 汉字, 教师在 教的时候一定要更加强调。

\section{3 三年级的错别字}

三年级错字类型统计结果

\begin{tabular}{|c|c|c|c|}
\hline \multicolumn{4}{|c|}{ 三年级 } \\
\hline 次数 & 写错的字 & 错的原因 & 错字类型 \\
\hline 8 & 侥 & “イ" 写成 “я" & 形近混淆 \\
\hline 7 & 壤 & 把下面的部分写成 “赛” & 形近混淆 \\
\hline 6 & 肆 & “聿” 上面多了一横 & 笔画增损 \\
\hline 6 & 捺 & 把 “示” 写成 “余” & 笔形失准 \\
\hline 6 & 颤 & “亶” 上面少了 “.” & 笔画增损 \\
\hline 5 & 瞻 & "詹” 的上面写成 “." & 笔形失准 \\
\hline
\end{tabular}


Jurnal Cakrawala Mandarin

Asosiasi Program Studi Mandarin Indonesia

Vol.4, No.2, Oktober 2020: P66-84

E-ISSN: $2579-4906$

\begin{tabular}{|c|c|c|c|}
\hline 5 & 跋 & 少了 “点” & 笔画增损 \\
\hline 5 & 拔 & 少了 “.”，变成 “友” & 笔画增损 \\
\hline 4 & 丧 & 下面的部分写成 “衣” & 形近混淆 \\
\hline 4 & 索 & 上面的部分多了一横 & 笔画增损 \\
\hline 4 & 骤 & 下面的部分写成 “家” & 形近混淆 \\
\hline 4 & 赋 & “武” 多了一擞 & 形近混淆 \\
\hline 4 & 凄 & “妻” 上面少了一横 & 笔画增损 \\
\hline 4 & 悸 & 把 “季” 写成 “李” & 形近混淆 \\
\hline 3 & 杂 & 把坚钩写成竖 & 笔形失准 \\
\hline 3 & 虔 & 把上面的部分写成 “.” & 笔形失准 \\
\hline 3 & 寡 & 把中间的部分写成 “真” & 形近混淆 \\
\hline
\end{tabular}

从上述的表我们可以得知三年级要掌握的汉字比一二年级复杂, 笔画也特别多, 使 三年的学生在听写的时候常常写错。三年级学生与一二年级不同, 三年级的错字主要原 因使形近混淆与笔画增损, 这跟三年学生学的汉字有关, 三年级学的汉字比一二年级多, 比一二年及复杂，因此在形近混淆与笔画增损这方面犯的错误比较高。

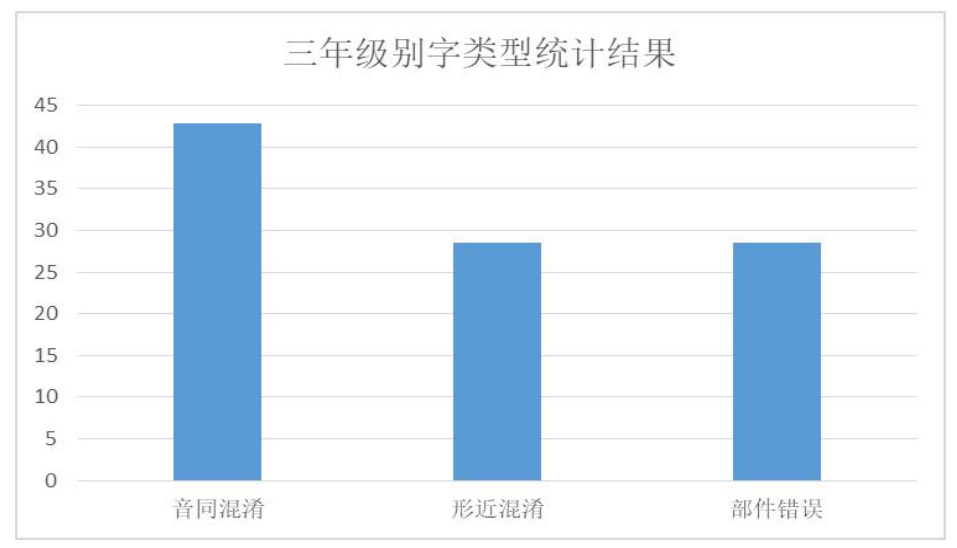

三年级学生的别字错误主要有音同混淆（6）, 部件错误（4）, 形近混淆（4）。 
三年级的学生错误的频率并不高, 澄、有、跋这三个字频率高一些其他的字基本上只有 两三个人错。

\section{四 结语}

根据本文的调查研究, 笔者可以得出如下结论:

1) 慈育大学中文系一至四年级学生对听写的兴趣还是很一般, 对教师使用的听写 方式的兴趣也是如此。学生已经习惯了教师的听写方式而认为教师的听写方式很单调, 不够多样化。虽然如此, 大多学生认为教师的一般听写方式已经符合他们理想的方式。 此外, 还有一些学生想尝试其他听写方式, 像教师进行一个有兴趣性的听写法或者教师 让学生自愿到黑板前听写。原因除了能够使听写不再单调, 还能够使学生在课堂上的表 现更积极。再者, 关于听写的时间, 大多学生认为教师应该在每次开始新的单元进行听 写一次。对该问题, 教师的做法已经符合了学生的要求, 因为学生最不喜欢的是教师突 然进行听写。

一至四年级学生大多认为听写是一个重要的课堂训练手段。对于大多学生来说, 听 写的作用是能够练习他们的汉字技巧。其他学生也认为通过听写, 他们能够练习语音的 技巧、展示语法点技巧、可以背生词增加词汇量以及训练记忆力。由此可见, 虽然学生 对听写的兴趣很一般, 但是听写确实值得进行的课堂训练手段。

2）学生在听写之前大多会提前准备。虽然大部分学生比较喜欢先等教师通知有听 写才准备, 但其他学生还会总是准备听写。这也可以看出从他们听写时的主要障碍。大 多认为汉字难以写, 难以背为听写时的主要障碍。因此, 他们很需要提前准备, 以至于 会取得好成绩。假如在听写时, 学生写不出来所听到的词语, 那大多是因为他们忘记怎 么写而很少是因为听不清楚教师念的词。学生对该问题的解决方法大多是会直接写拼 音, 还有其他学生也会写一个有同音的字。因此, 错别字经常出现在学生的听写结果。

3) 听写之后, 教师会改正学生的听写结果, 但是大多教师很少给学生解释他们写 错的字应该怎么写才正确。原因是因为教师已经在学生的书本上改正他们的错字。再者, 大多教师对学生的错字会总是给学生惩罚, 比如让他们再写几遍他们写错的字。大多教 师认为给学生惩罚是十分重要。原因是不仅能够训练他们的汉字书写能力, 而且能够避 免同样的错误再出现。对该问题, 学生也赞同教师的看法, 而且大部分学生也会完成教 师给的惩罚。 
4) 从一至四年级学生的听写结果, 学生最多出现的错别字以及学生出现的高频错 别字都是音同混淆的错别字。此外, 笔画增损、笔形失准、部件缺失是学生最多出现的 错别字。因此, 笔者发现有 3 个原因学生听写时会出现错别字现象, 依次为: 汉字本身 的难点、学生准备得不够充分、在听写时使他们容易听错写错, 因为听写是需要学生耳、 眼、手、脑并用。

\section{建议}

1) 对学生的建议

通过慈育大学中文系学生综合课听写现状的调查研究, 笔者发现有的学生在听写之 前的准备还不够充分。学生大多需要等教师的通知才会提前准备。其实, 作为学生要养 成良好的预习习惯。再者, 因为汉字本身的难点, 在准备听写时, 学生应该仔细识字, 关注汉字的音、形、义三个方面的内容, 尤其对一些比较难写的字。学生可以主动上网 查或者问其他同学正确的写法。此外, 正是识汉字的音形义, 学生才能避免错别字的出 现, 尤其是音同混淆的错别字类型, 如此学生还能提高自己的词汇量、记忆力。

在听写的时候, 因为各种各样的障碍, 学生应该保持专注, 不用紧张, 冷静听教师 念, 仔细地写汉字, 不要太快。有时候, 写得太快能导致学生出现笔形失准的错别字, 像把 “父” 写成 “文”、把 “竹” 写成像 “ $\mathrm{k}$ ”一样等等。假如写不出来, 学生可以先 写听到的词的拼音。假如以后想得出来, 才代替那个拼音写成正确的字。即便学生真的 忘了, 当教师批改之后他们的听写结果, 他们就会发现自己的错误, 是否只是忘了写, 或者是听错了。

教师批改之后听写结果, 无论有没有惩罚, 学生要主动看自己的听写结果, 发现自 己的错误并纠正。虽然有的学生认为教师给的惩罚不太重要甚至不重要, 但也要认真完 成。这也是养成一个良好的学习态度。

2) 对教师的建议

通过慈育大学中文系学生综合课听写现状的调查研究, 笔者发现每个教师的听写方 式有所不同。每个方式也有自己的优点和缺点。笔者认为, 最合理的听写方式是按常规 的听写方式, 教师念, 学生在听写本写。原因是因为教师能够了解每个学生的准备和能 力怎么样。此外, 每个学生也能够得到同样的机会, 顺便能取得成绩。只不过, 如果教 师一直如此进行听写, 可能学生也会觉得无聊, 因为听写方式不够多样化。因此, 笔者 希望教师有时候也会进行别的听写方式, 像让学生到黑板前听写、突然进行听写或者进 
行一个有兴趣性的听写方式, 比如听写比赛等等。让学生上黑板听写能提高学生的积极 性。听写完后学生可以一起纠正, 从其他同学的错别字学习。教师也可以顺便看清学生 写汉字时的笔画和笔顺, 然后解释正确的写法, 这样也能让学生更意识到该如何写好汉 字, 尤其是比较难写的字。只不过, 这种方式的缺点是并不是每个学生都能得到机会到 黑板前听写, 因为这种方式是靠学生的积极性和其充分准备。其次, 突然进行听写可以 执行如果教师已经讲完了课本上的生词为检查学生的复习情况。此外, 教师可以设计学 生已经学习的生词和语法为听写内容。再者, 有兴趣性的听写方式可以在有空的时候执 行的, 因为在综合课, 教师必须讲的内容比较多。以下是笔者对有兴趣性的听写方式的 建议:

(1) 以接龙的听写方式

老师先念一个生词让学生写在书本上, 然后让一个学生再念另一个生词, 大家 按照他念的写下来。那个学生再指着别的学生来念生词, 这种听写方式会持续直到 听写结束。

(2) “句子填空式”的听写

向学生解释, 他们会听一些句子, 但是在句子当中有些词教师会用“beep”声代 替一些本课需要学习的词语。学生必须按常规完成听写, 但要根据学习的生词选 择填空。这样学生能对词义有深一步的认识, 而且记忆比较深刻。

(3) 传递句子听写

每一纵排为一组, 教师念第一个句子给最前排的学生。学生写下听到的内容 立即用耳语把纸上的内容告诉后面的学生。第二位学生也写下他听到的内容并告 诉后面的学生, 而第一个学生又回到老师那儿听和写第二个句子。这样依次进行 下去到最后排的学生把所传的句子交给老师。传得最快最准的组获胜。如果每一 组做完了, 老师与大家一起纠正。

(4) 跑-读-说-听-写

这种方式可以化被动为主动, 同时包括了听、说、读、写四个技能的运用。 具体做法如下:

a. 准备一段短文分成六到八个句子, 分别写在 8 张纸。

b. 把那 8 张纸做成卡片一样, 外面随意分别写着 1 到 8 , 不要分次序, 然后把它 们贴到教室内八个不同的地方。 
c. 将学生分成两个人一组, 学生分开坐在教室各处。一个人负责跑到不同地方, 把看到的信息记住, 然后跑回组员身边轻声细语地说; 一个人负责坐在椅子上, 写下听到的内容。中间不准改变彼此的角色。

d. 如果一组完成了, 就把作品给老师看。老师请他们排出整段短文该有的顺序。

在给学生听写的时候, 教师可以对学生严肃一点, 像给学生念的次数, 保持在两次, 在念的时候, 尽量不用给学生提示。如果觉得很需要给提示的话, 教师可以说一下词语 的解释, 学生就写那个词语。相比之下, 假如在学生没有准备的情况下, 教师突然进行 听写就可以给提示。因为如果听写的内容是脱离具体语境的单个字词, 学生会无法确定 字、词之义或难以辨别字、词的声调而将实际存在于记忆中的字、词进行错误的选取和 输出。在这种情况下, 语义完整、明确的句子形式的提示能帮他们写下来所听到的词, 而且能提高对同音字、近音字的分辨率, 便于他们在长时记忆中做出较为迅速、准确的 选择和提取。

在批改学生的听写, 笔者希望教师更仔细检查, 因为笔者还发现有的学生不太仔细 写汉字, 但教师不够仔细而没有指出学生的错误。教师也可以改正学生写错的字, 以便 他们会更清楚自己的错误, 只不过, 教师也应该提出学生的错字, 给他们解释正确的写 法。从本调查结果, 可见学生写错的原因最多是因为音同混淆。教师在解释的时候可以 顺便提醒如何辨别同音字, 以便学生更理解生词的音、形、义这三个方面。此外, 教师 也应该给学生惩罚让他们重写错字。果真需要也可以让他们顺便写笔画。这种方法也是 为了避免同样的错误再出现。

\section{参考文献}

[1] 姜丽萍. 汉语作为第二语言课堂教学 [M] . 北京: 北京大学出版社, 2011, 213 .

[2] 崔永华, 杨寄洲. 对外汉语可以教学技巧 [M] . 北京: 北京语言大学出版社, 1997.

[3] 刘若云. 论对外汉语初级班的听写 [J] . 广州: 中山大学学报论丛, 2006 (11)，200.

[4] 张辉, 杨楠. 汉语综合课教学法 [M] . 北京: 北京语言大学出版社, 2006, 196-198.

[5] 王静. 记忆原理对汉字听写训练的启示 [J] . 北京: 语言大学与研究, 2001 (1)，23.

[6] 孙德金. 对外汉字教学研究 [M] . 北京: 商务印书馆出版社, 2006, 446.

[7] 朱志平, 冯马萍. 汉语作为第二语言习得研究 [M] . 北京: 北京师范大学出版社, 2014, 69-70. 
[8] 霍丽暖. 作为形成性评价手段的听写法在对外汉语初级课堂中的应用研究 [D]. 北京: 北京外 国语大学, 2014.

[9] 商海静. 小学高年级学生错别字现象的调查研究 [D] . 济南: 北京师范大学出版社, 2012.

\section{Biografi （作者简介）}

$\begin{array}{ll}\text { Name } & \text { : Kumala Dewi (朱小玲) } \\ \text { Country } & \text { : Indonesia (印度尼西亚) } \\ \text { University } & \text { : Bunda Mulia (慈育大学) } \\ \text { Hp } & \text { : 087788893897 } \\ \text { Email } & \text { : kdewi@bundamulia.ac.id }\end{array}$

$\begin{array}{ll}\text { Name } & \text { : Siska (蔡静怡) } \\ \text { Country } & \text { : Indonesia (印度尼西亚) } \\ \text { University } & : \text { Bunda Mulia (慈育大学) } \\ \text { Hp } & \text { : 087832655141 } \\ \text { Email } & \text { : timotiuskiranakarya@yahoo.com }\end{array}$

